\title{
CLUSTER MODEL OF CHARGE TRANSFER EXCITONS IN MOLECULAR CRYSTALS. I. NEAT CRYSTALS*
}

\author{
P. Petelenz and Á. Eilmes \\ K. Guminski Department of Theoretical Chemistry, Jagellonian University \\ M. Karasia 3, 30-060 Kraków, Poland \\ (Received Seplember 27, 1990)
}

\begin{abstract}
A two-dimensional finite cluster model is proposed to describe the coupling of low energy charge transfer and Frenkel states of an anthracene-like molecular crystal. The results confirm most of the qualitative conclusions of the extensively used linear crystal model, but suggest the necessity to review some of its quantitative results. Potential usefulness of the cluster model for the description of doped crystals is pointed out.
\end{abstract}

PACS numbers:

\section{Introduction}

The application of electroabsorption spectroscopy for the investigation of charge transfer (CT) excitons in molecular crystals [1,2] stimulated a renewed interest in the theoretical description of such states. This description has so far been based on two essentially complementary models.

On the one hand, there has been the localized approach where a charges are envisaged as stationary, the hole residing at one and the electron at another lattice site. In that case, the energies of the CT states follow from purely electrostatic considerations [3-8]. Their calculation, although tedious [3], can be greatly facilitated by the Fourier transformation [4-8]. The calculations can be performed with due account taken of the complete three-dimensional structure of the crystal.

On the other hand, in the alternative approach initiated by Merrifield [9-11], the structure of the crystal has to be greatly simplified: the crystal is represented just by a one-dimensional array of equally spaced molecules. This is the price that

*This work was supported by a grant-in-aid from the Jagellonian University. 
has to be paid for the inclusion of the off-diagonal (in the local representation) matrix elements of the Hamiltonian which are responsible for the delocalization of the charges over many crystal sites, and are neglected in the localized approach.

As the off-diagonal matrix elements of the Hamiltonian (CT integrals) are at least linear in (small) intermolecular overlap integrals, the CT state energy values resulting from the localized approach are correct to about $0.1 \mathrm{eV}$ and provide a very reasonable starting point for a more detailed model taking into account the dynamic effects giving rise to charge delocalization. This observation motivated the generalization $[8,12-16]$ of the Merrifield model for those molecular crystals which, like anthracene, contain two molecules in the unit cell.

Arguments based on the analysis of the values of the CT integrals [15] led to the tentative conclusion that the linear model with the modification mentioned above is likely to account very well for most properties of the low energy CT states in anthracene-like crystals. In fact, the CT interaction between the molecules belonging to different $a b$ planes of such crystals is much weaker than the corresponding interaction along the $b$ axis. Since the CT interactions along the $a$ axis are equally weak, the crystal, from the point of view of its lowest CT states, can be split into a set of linear crystals with two molecules in the unit cell and the stacking axis directed along the $b$ axis of the actual three dimensional crystal. The expected cancellation of errors of different origins [15] provided a reasonable basis for the application of the linear model for the interpretation $[15,16]$ of the experimental results $[1,2]$.

The aspect of the linear model which has barely been considered [16] is due to the fact that the $a b$ plane of an anthracene like crystal can be partitioned into the linear crystals in two different ways, so that a given molecule belongs either to the first or to the second sublattice. These two partitions produce two physically distinct sets of CT states of $(1 / 2,1 / 2,0)$ parentage (Fig. 1), and the linear model can deal with only one of them at a time. An attempt to account for this fact was made in Ref.[16] where the two subsets of the $(1 / 2,1 / 2,0)$ states were assumed to be degenerate and the total electroabsorption signal was calculated with an additional weighting factor of 2 . Ilowever, the results obtained in this way could be only qualitatively correct. In fact, although the direct coupling between the two sets of CT states is negligible due to the very small values of the CT integrals for the $(1,0,0)$ direction, there is an indirect interaction via the CT states of $(0,1,0)$ parentage, mediated by the CT integrals corresponding to the $(1 / 2,1 / 2,0)$ direction. This interaction is bound to split the (otherwise degenerate) states of $(1 / 2,1 / 2,0)$ parentage originating from the two different partitions of the $a b$ plane into linear crystals.

A one-dimensional model is inherently incapable of dealing with this splitting and a two dimensional model becomes necessary. While a complete study of an infinite two-dimensional crystal presents interest in itself, a finite cluster approach offers potential advantagès for future use in the description of doped crystals where the translational symmetry of the lattice is locally broken. With that aim in mind, in this paper we present a finite two-dimensional cluster model with periodic boundary conditions. For not too large clusters, the Hamiltonian (based on the same kind of approximations as have been used in the one-dimensional 


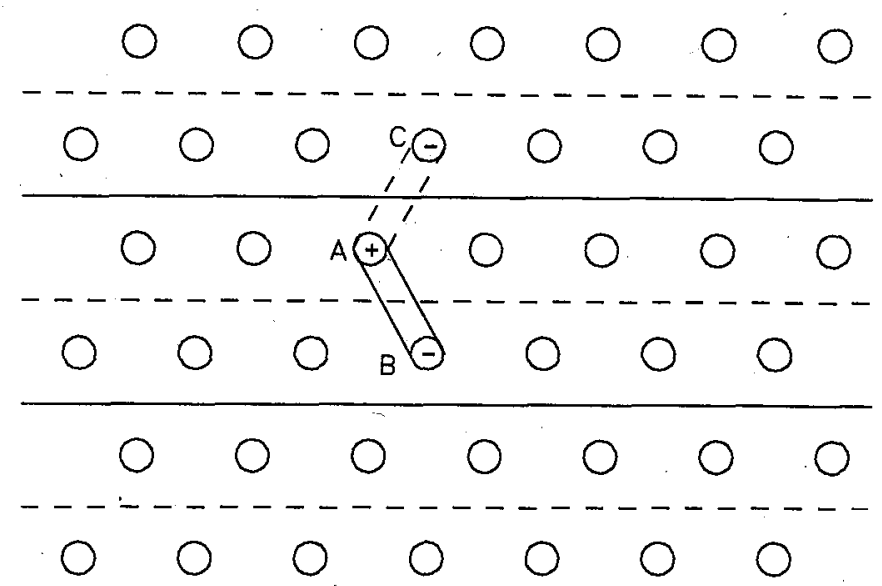

Fig. 1. Alternative ways of partitioning a planar crystal into linear crystals with two molecules in the unit cell. If the first way of partitioning is adopted (solid lines) the $\mathrm{A}^{+} \mathrm{C}^{-}$state is disregarded, while with the second way of partitioning (broken lines) the $\mathrm{A}^{+} \mathrm{B}^{-}$state is disregarded.

models) can be diagonalized numerically, yielding the eigenvalues and eigenvectors.

Due to the periodic boundary conditions, the crystal momentum $k$ is still a good quantum number, like in an infinite crystal. Consequently, the cluster is essentially equivalent to a two dimesional crystal where only some specific values of crystal momentum $k$ are allowed (rather than a quasicontinuum). Whatever the size of the cluster, $\mathrm{k}=(0,0)$ is always one of those values. By a judicious choice of the cluster size (videinfra) it is possible to ensure thiat the values of $k=(0, \pm \pi)$ have been allowed as well.

The energy values of the states which correspond to $k=(0,0)$ and $k=(0, \pm \pi)$ do not depend on the size of the cluster, so that relatively small clusters may be used in actual calculations. As just these energy values are the most interesting ones from the point of view of spectroscopic applications [15, 16], the planar cluster model seems to provide a feasible way to test the validity of the linear crystal model used previously [14-16].

\section{The cluster model}

Let us consider a rigid planar cluster consisting of $n \times m$ molecules from the $a b$ plane of an anthracene-like crystal ( $n$ molecules along the $b$ axis and $m$ molecules along the $a$ axis). In order to imitate the siluation in the bulk of the crystal and to eliminate artifacts due to the presence of boundaries, we impose the periodic boundary conditions (Fig. 2). In other words, we join the left edge of the cluster with its right edge and the upper edge with the lower, so that the topology of the cluster becomes equivalent to that of a torus. 


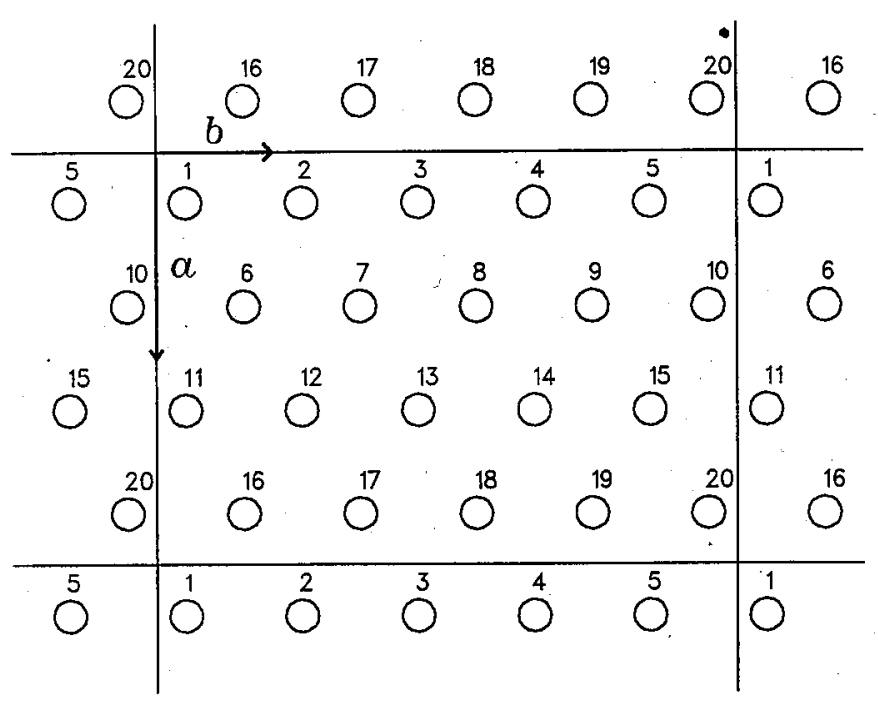

Fig. 2. The model two-dimensional cluster (within the solid lines) with periodic boundary conditions.

Each molecule in the cluster has six nearest neighbours. The four positions $( \pm 1 / 2, \pm 1 / 2,0)$ are equivalent, and so are the two positions $(0, \pm 1,0)$. (Note that the $( \pm 1 / 2, \pm 1 / 2,0)$ positions are not equivalent to the $(0, \pm 1,0)$ positions $)$.

The cluster contains $N=m n$ molecules. In analogy to the linear model [14-16] we confine the treatment to the nearest neighbour CT states, so that our basis consists of the following localized states: i) the set of $N$ functions describing Frenkel excitons located at each of the $N$ molecules. These functions will be denoted as $\left|A^{*}\right\rangle$, where $A$ stands for the molecule in hand. Only one Frenkel state per molecule is taken into account; ii) the set of $6 N$ functions (denoted as $\left|A^{+} B^{-}\right\rangle$) describing the CT states with the hole located at a given molecule $A$ and the electron located at one of the six nearest neighbours $B .4 N$ functions represent the $(1 / 2,1 / 2,0)$ states and $2 N$ functions represent the $(0,1,0)$ states (the "crosswise" and "lengthwise" CT states of Refs. [12,15], respectively). In this basis the matrix elements of the Hamiltonian read:

$\left\langle A^{*}|H| A^{*}\right\rangle=E^{\mathrm{F}}$ is the diagonal energy of the Frenkel exciton located at molecule $A$;

$\left\langle A^{+} B^{-}|H| A^{+} B^{-}\right\rangle= \begin{cases}E^{\mathrm{P}} & \begin{array}{l}\text { if the molecule } B \text { is the nearest neighbour of the } \\ \text { molecule } A \text { in one of the }( \pm 1 / 2, \pm 1 / 2,0) \text { directions }\end{array} \\ E^{\mathrm{L}} & \begin{array}{l}\text { if the molecule } B \text { is the nearest neighbour of the } \\ \text { molecule } A \text { in the }(0, \pm 1,0) \text { direction }\end{array}\end{cases}$ 
are the electrostatic energies of the CT states ("crosswise" and "lengthwise", respectively);

$\left\langle A^{*} B|H| A B^{*}\right\rangle= \begin{cases}M & \begin{array}{l}\text { if the molecule } B \text { is the nearest neighbour of the } \\ \text { molecule } A \text { in one of the }( \pm 1 / 2, \pm 1 / 2,0) \text { directions }\end{array} \\ W & \begin{array}{l}\text { if the molecule } B \text { is the nearest neighbour of the } \\ \text { molecule } A \text { in the }(0, \pm 1,0) \text { direction }\end{array}\end{cases}$

are the Frenkel exciton transfer ("resonance") integrals for the $(1 / 2,1 / 2,0)$ and $(0,1,0)$ direction, respectively;

$\left\langle A^{+} B^{-}|H| A^{+} C^{-}\right\rangle= \begin{cases}J_{\mathrm{e}}^{\prime} & \begin{array}{l}\text { if the molecule } C \text { is the nearest neighbour of the } \\ \text { molecule } B \text { in one of the }( \pm 1 / 2, \pm+1 / 2,0) \text { directions }\end{array} \\ J_{\mathrm{e}} & \begin{array}{l}\text { if the molecule } C \text { is the nearest neighbour of the } \\ \text { molecule } B \text { in the }(0, \pm 1,0) \text { direction }\end{array}\end{cases}$

$\left\langle A^{+} B^{-}|H| C^{+} B^{-}\right\rangle= \begin{cases}J_{\mathrm{h}}^{\prime} & \begin{array}{l}\text { if the molecule } C \text { is the nearest neighbour of the } \\ \text { molecule } A \text { in one of the }( \pm 1 / 2, \pm 1 / 2,0) \text { directions }\end{array} \\ J_{\mathrm{h}} & \begin{array}{l}\text { if the molecule } C \text { is the nearest neighbour of the } \\ \text { molecule } A \text { in the }(0, \pm 1,0) \text { direction }\end{array}\end{cases}$

are the corresponding electron and hole transfer integrals, respectively; and

$\left\langle A^{*} B|H| A^{+} B^{-}\right\rangle= \begin{cases}D_{\mathrm{e}}^{\prime} & \begin{array}{l}\text { if the molecule } B \text { is the nearest neighbour of the } \\ \text { molecule } A \text { in one of the }( \pm 1 / 2, \pm 1 / 2,0) \text { directions }\end{array} \\ D_{\mathrm{e}} & \begin{array}{l}\text { if the molecule } B \text { is the nearest neighbour of the } \\ \text { molecule } A \text { in the }(0, \pm 1,0) \text { direction }\end{array}\end{cases}$

$\left\langle A^{*} B|H| A^{-} B^{+}\right\rangle= \begin{cases}D_{\mathrm{h}}^{\prime} & \begin{array}{l}\text { if the molecule } B \text { is the nearest neighbour of the } \\ \text { molecule } A \text { in one of the }( \pm 1 / 2, \pm 1 / 2,0) \text { directions }\end{array} \\ D_{\mathrm{h}} & \begin{array}{l}\text { if the molecule } B \text { is the nearest neighbour of the } \\ \text { molecule } A \text { in the }(0, \pm 1,0) \text { direction }\end{array}\end{cases}$

are the Frenkel exciton dissociation integrals.

All other matrix elements are set equal to zero.

The above labelling of the matrix elements follows directly from that used for the one-dimensional model of Refs.[15, 16], where the definitions in terms of molecular orbitals as well as other details may be found. 
To all intents of the present paper (which is merely to test the one-dimensional model), vibrational degrees of freedom are irrelevant and are consistently disregarded.

\section{Implementation and input data}

In the actual implementation we use, the basis functions are numbered in the following way:

The first $N$ functions are those corresponding to Frenkel excitons and they are labelled by the number of the molecule on which the exciton resides. The following $6 N$ functions represent the CT states. As each molecule has six nearest neighbours, there are six different CT states with the hole located at a given molecule and the electron at a nearest neighbour molecule. The direction of a vector pointing from the position of the hole to that of the electron is labelled by an integer $l$, running from 1 for $(1 / 2,1 / 2,0)$ to 6 for $(1 / 2,-1 / 2,0)$. The basis function corresponding to the CT state with the hole located at $n$-th molecule and the electron located at its nearest neighbour in the direction $l$ has the number $N+6(n-1)+l$.

The computer routine building the Hamiltonian matrix scans all the molecules of the cluster and for each molecule all the directions. Given the number of the molecule and of the direction, another routine finds the number of the corresponding nearest neighbour molecule and subsequently the numbers of the basis functions representing the $\mathrm{CT}$ states involving the two molecules. This set of data allows to define all the necessary matrix elements according to the prescriptions of the preceding section.

The objective of this paper is to test the performance of the one-dimensional model used previously rather than to describe any specific physical system. Consequently, the input data need not represent any actual crystal. Iowever, to make sure that the conclusions pertain to a physically relevant situation, we have used the input data corresponding to the anthracene crystal which was the standard testing case for previous theories $[1,2,7,8,14-18]$. The numerical values of the parameters are as follows (all values in $\mathrm{eV}$ ): $E^{\mathbf{F}}=3.2234, E^{\mathbf{P}}=3.562, E^{\mathbf{L}}=$ $3.685, W=-0.0479, M=-0.0152, J_{\mathrm{e}}=0.0244, J_{\mathrm{h}}=-0.0443, J_{\mathrm{e}}^{\prime}=0.0526$, $J_{\mathrm{h}}^{\prime}=-0.0418, D_{\mathrm{e}}=0.0281, D_{\mathrm{h}}=-0.0510, D_{\mathrm{e}}^{\prime}=0.0605, D_{\mathrm{h}}^{\prime}=-0.0481$.

They are essentially the same as in the most recent paper on anthracene [18].

In view of the continuing discussion in the literature [3, 4, 15-19] it was not clear whether the band gap $E^{\mathrm{G}}=4.42 \mathrm{eV}$ or $E^{\mathrm{G}}=4.25 \mathrm{eV}$ would be appropriate for the anthracene crystal. Due to the model nature of the present paper, this issue is of secondary importance here. Therefore, in order to avoid the problems of vibronic coupling [16], irrelevant in the present context, we have used the former, more conservative value.

This paper is meant to answer the specific question how wcll the results of the linear model can reproduce those obtained from the planar model and to what extent the discrepancies can be reduced by a judicious renormalization of the parameters. It was argued in [18] that some effects of the interactions which are not explicitly included in the linear model can be accounted for by treating some 
parameters as effective. The proposed refinements, aimed to improve the physical relevance of the results for the Frenkel states, consisted in [18]: a) doubling of the Frenkel exciton transfer integral $M$ to account for the resonance interaction with the nearest neighbour molecules which are not included in the model linear crystal and

b) renormalization of the diagonal Frenkel exciton energy

$$
E^{\mathbf{F}} \rightarrow E^{F^{\prime}}=E^{\mathbf{F}}-2\left(D_{\mathbf{e}}^{\prime 2}+D_{h}^{\prime 2}\right) /\left(E^{\mathbf{P}}-E^{\mathbf{F}}\right)
$$

to account for the leading part of the CT interaction with those molecules.

As an alternative to b), we presently propose:

c) renormalization of the CT and exciton dissociation integrals $J_{\mathbf{e}}^{\prime}, J_{\mathrm{h}}^{\prime}, D_{\mathbf{e}}^{\prime}, D_{\mathrm{h}}^{\prime}$ by the factor of $2^{1 / 2}$.

In the following, we will compare the results of the planar model, obtained by numerical diagonalization of the IIamiltonian matrix of Section 2, with two alternative sets of results of the linear model. The two sets were calculated with the input parameters adjusted according to a) and b) (Linear Model 1, hereafter referred to as LM1), and to a) and c) (Linear Model $2=$ LM2), respectively.

For the linear model calculations, the method of Ref. [17] (to which the Reader is referred for details) was used. (In Eq.(6d) of Ref.[17], $D_{\mathrm{h}} \sin \left(k n^{\prime}\right)$ should be replaced by $-D_{\mathrm{h}} \sin (k n)$, and the sine term should be included in the sum over $n$. The error does not affect the results at $k=0$ and $k=\pi$, but slightly changes some of the dispersion relations at other $k$ values).

The cluster type calculations (Section 2) have been performed for clusters differing both in "length" $(n=3-6)$ and in "width" $(m=4-8)$. In the pseudo-band calculations of the linear model, the "length" (number of unit cells) of the model crystal was assumed to be the same as the "length" $n$ of the corresponding cluster, so that the energy values were calculated only at the $n$ values of $k_{\mathrm{b}}$ permitted by the periodic boundary conditions. This allowed to refer the eigenvalues obtained from the cluster model to pseudo-band energies at specific points of the Brillouin zone in the one-dimensional model, and facilitated the analysis of the results.

It is worth noting that a pseudo-band energy eigenvalue for a given value of $k_{\mathrm{b}}$ does not depend on the "length" $n$ of the model linear crystal for which it was calculated. Therefore, the differences between the results obtained for different $n$ values are due exclusively to the fact that different sets of $k_{\mathrm{b}}$ values are allowed. This also means that the obtained eigenvalues are exactly the same as for an infinite crystal (at the same value of crystal momentum).

This observation allowed us to ascribe the cluster eigenvalues to specific $k$ values without the necessity to invoke and analyze the eigenvectors. For example, with $n=3,4$ and 6 , the sets of allowed $k_{\mathrm{b}}$ values are (in units of $\pi$ ) $\{-2 / 3,0,2 / 3\} ;\{-1,-1 / 2,0,1 / 2\}$ and $\{-1,-2 / 3,-1 / 3,0,1 / 3,2 / 3\}$, respectively. Accordingly, the eigenvalues that coincide for $n=3,4$ and 6 correspond to $k_{\mathrm{b}}=0$, those that coincide for $n=4$ and 6 correspond to $k_{\mathrm{b}}=-\pi$, etc.

Some of the $k_{\mathrm{b}}=0$ states are easy to identify anyway due to the absence of the $(k,-k)$ degeneracy resulting from the time inversion symmetry. As for centrosymmetric crystals the electronic states are degenerate at the $k_{\mathrm{b}}=-\pi$ edge 
of the Brillouin zone [16], non-degenerate states may appear only at $k_{\mathrm{b}}=0$. Therefore, lack of degeneracy is an unambiguous indication that the state in hand corresponds to the centre of the Brillouin zone, but there may still be some states which are degenerate even at $k_{\mathrm{b}}=0$, which would not be detected by this criterion.

An analogous argument may be used for $k_{\mathrm{a}}$.

\section{Results and discussion}

The main quantities of direct spectroscopic interest are the energy eigenvalues in the centre $\left(k_{\mathrm{b}}=k_{\mathrm{a}}=0\right)$ [15] and at the $k_{\mathrm{b}}= \pm \pi\left(k_{\mathrm{a}}=0\right)$ edges of the Brillouin zone [16]. Their values obtained from the planar cluster model are compared in Table I with those from pseudo-band calculations (LM1). As it has been explained in Section 2, some of the CT states of $(1 / 2,1 / 2,0)$ parentage are not included in the linear model, which is reflected in the table. Apart from this difference, it is readily seen that the results of Linear Model 1 are always qualitatively and in some aspects even quantitatively correct.

\section{TABLE I}

Energy values of Frenkel and CT excilons at the centre and at the edge of the Brillouin zone. All values in $\mathrm{eV}$

\begin{tabular}{l|c|c|l|l}
\hline \hline State & $\begin{array}{c}\text { Linear } \\
k_{\mathrm{b}}=0\end{array}$ & $\begin{array}{l}\text { Model I } \\
k_{\mathrm{b}}=\pi\end{array}$ & $\begin{array}{l}\text { Cluster } \\
k_{\mathrm{b}}=0\end{array}$ & $\begin{array}{l}\text { Model } \\
k_{\mathrm{b}}=\pi\end{array}$ \\
\hline Frenkel & 3.060 & 3.221 & 3.049 & 3.218 \\
excitons & 3.067 & 3.221 & 3.094 & 3.218 \\
\hline & 3.526 & 3.497 & 3.490 & $3.493^{*}$ \\
CT $\left[\frac{1}{2} \frac{1}{2} 0\right]$ & 3.542 & 3.497 & $3.5421^{*}$ & $3.493^{*}$ \\
& 3.548 & 3.587 & $3.5421^{*}$ & 3.499 \\
states & 3.581 & 3.587 & 3.5422 & 3.499 \\
& & & 3.557 & 3.567 \\
& & & 3.580 & 3.567 \\
& & & $3.582^{*}$ & $3.630^{*}$ \\
& & & $3.582^{*}$ & $3.630^{*}$ \\
\hline CT (0 1 0) & 3.686 & 3.708 & 3.687 & 3.725 \\
states & 3.687 & 3.708 & 3.688 & 3.725 \\
& 3.735 & 3.765 & 3.777 & 3.804 \\
& 3.741 & 3.765 & 3.779 & 3.804
\end{tabular}

The main discrepancies occur in the manifold of the states of $(0,1,0)$ parentage, whose energies are consistently underestimated by the linear model. The Davydov splitting of the Frenkel states is also seriously underestimated, but this effect is probably due to an accidental cancellation of different contributions for this particular parameter set.

It is interesting to note that most of the discrepancies can be removed by readjusting the parameters used in the linear model. With the renormalized values of the parameters (LM2), the linear model reproduces, correct to less than $1 \mathrm{meV}$, all the energy values resulting from the cluster model calculations, except those 
indicated in Table $\mathrm{I}$ by asterisks. The latter ones represent the four CT states of $(1 / 2,1 / 2,0)$ parentage which are not included in the linear model and therefore the fact that they cannot be handled is an inherent limitation of that model. They are doubly degenerate both at the centre and at the edge of the Brilloun zone.

These observations support the qualitative conclusions of Refs.[14-18], but refute some of the quantitative results. They suggest that the energy values of CT transitions calculated in $[15,16,20]$ have to be re-evaluated. At least to some extent this might be done within the linear crystal model with the parameters renormalized according to the prescription of LM2. This approach may be particularly useful for the calculations of the pressure dependence of Frenkel exciton energies [18].

One of the problems where a model of CT states in polyacene crystals was needed concerned the interpretation of electro-absorption spectra [1, 2]. Several models have been introduced in that context (their results for the states of $(1 / 2,1 / 2,0)$ and $(0,1,0)$ parentage are visualized in Fig. 3$)$ :

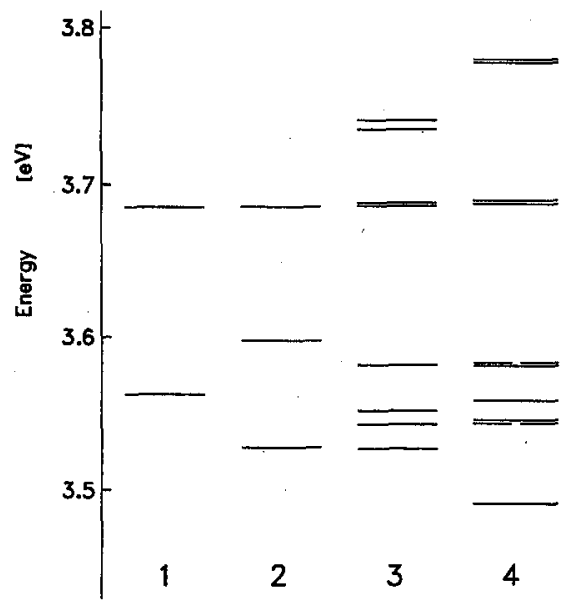

Fig. 3. Models of CT states in polyacene crystals (only the states of $(1 / 2,1 / 2,0)$ and $(0,1,0)$ parentage are shown): (1.) localized model; $(2$.$) its ad hoc extension of Ref.[8];$ (3.) linear model of Ref.[14] ( $k=0$ states); (4.) planar model ( $k=0$ states obtained from cluster calculation of this paper). All energies calculated for the set of data described in the lext.

1. the reasonable starting point $[1,2]$ was the localized model where the interactions mediated by the off-diagonal (in the local basis) matrix elements of the Hamiltonian were totally disregarded. As these elements are known to be of the same order of magnitude as the gaps between the diagonal energies, this approach was conceptually unsatisfactory. It was also inherently incompatible with experimentally observed intensities [8]; 

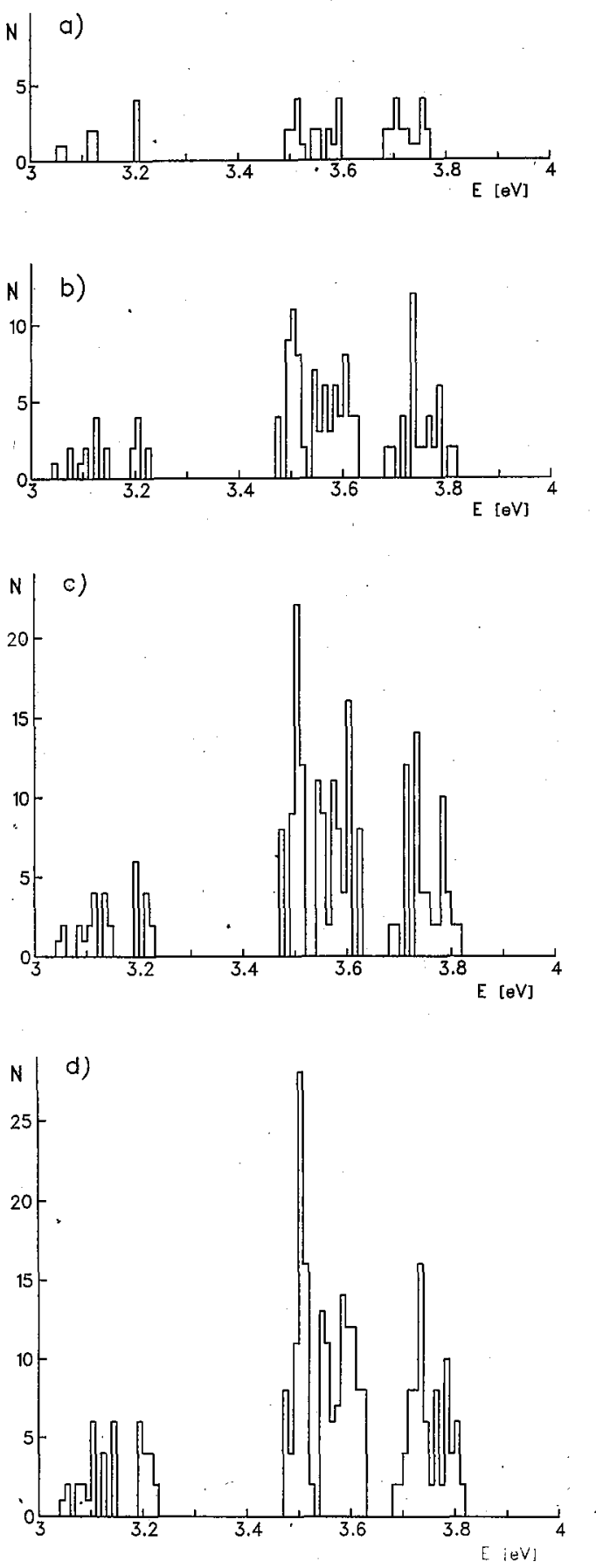

Fig. 4. Number of energy eigenstates per energy interval for varying "width" of the two-dimensional cluster ( $m=2(\mathrm{a}), 4(\mathrm{~b}), 6(\mathrm{c})$ and $8(\mathrm{~d})$, respectively, and $n=5)$. 
2. the localized model was supplemented by an ad hoc inclusion of the additional splitting due to the electron and hole transfer integrals [8]. This allowed to rationalize the observed intensities in qualitative terms, but offered no way of systematic verification by direct calculations;

3. a linear crystal model was introduced [14], which, although very approximate, provided a more or less systematic framework to calculate both the energies and the intensities of CT transitions.

Subsequent analysis [15] demonstrated that only degenerate CT states can produce the observed [2] linear Stark effect. Still within the same linear model of Ref.[14], the involvement of the states from the edge of the Brillouin zonc (accompanied by phonons) was postulated, with ensuing proposal regarding the value of the band gap [15].

The presence of the additional states of $(1 / 2,1 / 2,0)$ parentage discussed in this paper suggests that a reinterpretation of electro-absorption spectra [1,2] may again become necessary. The fact that they are degenerate may have important consequences for the final interpretation, possibly eliminating the necessity to invoke the phonons. The new states should also be included in the band structure calculations [17].

Another quantity of potential relevance to the interpretation of experimental results is the density of states [16]. Obviously, it should differ for one- and two-dimensional models. This aspect of our results is presented in Fig.4 in the form of histograms representing the number of cluster eigenstates per energy interval, depending on the "width" $m$ of the cluster (the "length" of the cluster is fixed at $n=5$ ). (The figure should be considered only as an illustration of the trend - the actual values obtained for any finite cluster are of course an extremely poor appioximation of the density of states in an infinite crystal).

The clusters under consideration may be thought of as one, two, three or four linear crystals with two molecules in the unit sell, combined together.

The histograms show that apart from the trivial increase of the total number of states, their distribution in energy also changes with the change of cluster size. The most conspicuous change takes place when the cluster width increases from two to four molecules, the former case representing the linear crystal with two molecules in the unit cell [14-18]. This is the expected effect of the change of topology. In other cases, the changes are present, but not so pronounced.

The observation that for truly two-dimensional clusters $(m=4 \div 8)$ the general distribution of energy levels does not depend very strongly on cluster size suggests that even small clusters may provide meaningful conclusions and give interesting insight into the physics of the problem. This conclusion may have some practical consequences.

In fact, the cluster approach seems to be particularly well suited for the consideration of doped crystals, where the presence of an impurity breaks the translational symmetry of the latlice so that the local description becomes appropriate. The results presented above suggest that relatively small clusters may suffice to draw meaningful conclusions in that case. This problem will be taken up in a forthcoming paper, dealing specifically with doped crystals and aimed to interpret the recent experimental data [21-23]. We hope that the cluster model 
will provide a basis for a future approach formulated in terms of Green's functions.

\section{References}

[1] L. Sebastian, G. Weiser, G. Peter, H. Bässler, Chem. Phys. 61, 125 (1981).

[2] L. Sebastian, G. Weiser, II. Bässler, Chem. Phys. 75, 103 (1983).

[3] A. Jurgis, E.A. Silinsh, Phys. Status Solidi53 B, 735 (1972);

E.A. Silinsh, Organic molecular crystals: Their electronic states, Springer, Berlin 1980.

[4] R.W. Munn, Mol. Phys. 64, 1 (1988).

[5] P.J. Bounds, R.W. Munn, Chem. Phys. 59, 41 (1981); 44, 103 (1979); 59, 47 (1981).

[6] P.J. Bounds, W. Siebrand, Chem. Phys.Lelt. 75, 414 (1980).

[7] P.J. Bounds, P. Petelenz, W. Siebrand, Chem. Phys. 63, 303 (1981).

[8] P.J. Bounds, W. Siebrand, I. Eisenstein, R.W. Munn, P. Petelenz, Chem. Phys. 95, 197 (1985).

[9] R.E. Merrificld, J. Chem. Phys. 34, 1835 (1961).

[10] J.P. Hernandez, S.I. Choi, J. Chem. Phys. 50, 1524 (1969).

[11] W.L. Pollans, S.I.Choi, J. Chem. Phys. 52, 3691 (1970).

[12] P. Petelenz, Phys. Stalus Solidi 78 B, (1976) 489; 79, 61 (1977).

[13] P. Reineker, B.J. Schmid, Phys. Status Solidi 124, 657 (1984);

B.J. Schmid, Thesis, University of UIm (1985).

[14] P. Petelenz, in Proceedings of the XI Molecular Crystal Symposium, Lugano 1985, p.230.

[15] R.W. Munn, P. Petelenz, W. Siebrand, Chem. Phys. 111, 207 (1987).

[16] P. Petelenz, V.H. Smith, Jr., Chem. Phys. 131, 409 (1989).

[17] P. Petelenz, Chem. Phys. 133, 199 (1989).

[18] P. Petclenz, Chem. Phys. 138, 35 (1989).

[19] N. Sato, H. Inokuchi, E.A. Silinsh, Chem. Phys. 115, 269 (1987).

[20] B. Petelenz, P. Petelenz, H.F. Shurvell, V.II. Smith, Jr., Chem. Phys. 119,25 (1988).

[21] P. Petelenz, I.V. Brovchenko, D. Mucha, M. Slawik, Chem. Phys. 143, 415 (1990).

[22] I.V. Brovchenko, N.I. Ostapenko, V.I. Sugakov, M.T. Shpak, Almos. Ocean. Phys. Izv. Akad. Nauk SSSR 52, 659 (1988).

[23] I.V. Brovchenko, N.I. Ostapenko, M.T. Shpak, Phys. Solis Earth Fiz. Tverd. Tela 30, 261 (1988). 